\title{
Buenas prácticas de dispensación en dosis unitaria del Hospital Regional Zacarias Correa Valdivia de Huancavelica
}

\author{
Good dispensing practices per unit dose at the Zacarías Correa Valdivia Regional Hospital in Huancavelica
}

Mery Ñahui Paitan 1,a, Condi Paola Meza Santivañez ${ }^{1, a}$, Lizzy Jeanette Mendoza Gutierrez ${ }^{1, b}$

\section{RESUMEN}

Objetivo: Determinar las buenas prácticas de dispensación por dosis unitaria en el Hospital Regional Zacarías Correa Valdivia de Huancavelica. Material y métodos: Metodología científica, con enfoque básico, nivel descriptivo y de diseño no experimental, transversal y descriptivo. La muestra estuvo conformada por 11 profesionales químicos farmacéuticos del HRZCVH. Se utilizó un cuestionario "BPDDUMP”, el cual fue diseñado y validado por juicio de expertos. Resultados: Del total de profesionales del HRZCVH, mayor a la mitad (54.55\%) de Q.M., confirman que la prescripción no cumple con los datos requeridos, tienen dificultades para comprender la receta médica, e indican que las recetas médicas no tienen letra legible y clara. El $36.36 \%$ de Q. F. no actualizan el perfil farmacoterapeutico y el $54.55 \%$ no realiza la verificación y seguimiento de la ubicación del paciente. El 45.55\% de los Q. F., indican que los cajetines de la unidosis, no están correctamente identificados y el $18.18 \%$ no revisan y corroboran la medicación depositada en cada cajetín. El $27.27 \%$ de Q. F., indican que la dosis preparada de un paciente, ha sido insuficiente en 24 horas. $\mathrm{Y}$ el $54.55 \%$ han notado insuficiencia de los productos farmacéuticos que en una urgencia y/o emergencia, y solo el $36.36 \%$ de Q. F. cuentan con un cajetín de medicamentos u otros productos de reserva por cada paciente. El 27.27 \% de Q.F. confirman que no existe un control estricto a la hora de la entrega, de los medicamentos y demás productos; y el $36.36 \%$, para estupefacientes. El $36.36 \%$ de Q.F. confirman que la administración del medicamento no se da en el horario exacto. Confirmando por el $54.55 \%$, quienes han observado dificultades en el cumplimiento de la hora de administración de las unidosis, ratificando con el $63.64 \%$ que no observa el cumplimiento estricto, en cuanto la frecuencia de administración del medicamento a cada paciente. Conclusiones: Se verifico la existencia de dificultades en el proceso de dispensación por dosis unitaria según al cumplimiento de la Norma técnica del Ministerio de Salud. Lo que limita las buenas prácticas de dispensación por dosis unitaria en el Hospital Regional Zacarías Correa Valdivia de Huancavelica.

PALABRAS CLAVE: Químico Farmacéutico, Buenas prácticas, Dispensación y Dosis unitaria.

\section{SUMMARY}

Objective: To determine the good dispensing practices per unit dose at the Zacarías Correa Valdivia Regional Hospital in Huancavelica (HRZCVH). Material and Methods: Scientific methodology, with a basic approach, descriptive level and non-experimental, transversal and descriptive design. The sample consisted of 11 pharmaceutical professionals from HRZCVH. A "BPDDUMP" questionnaire was used, which was designed and validated by expert judgment. Results: Of the total of HRZCVH professionals, more than half (54.55\%) of QM,

Facultad de Ciencias de la Salud, Universidad Privada De Huancayo Franklin Roosevelt. Huancayo, Perú.

a Bachiller; ${ }^{\text {b Docente, Mg. Q.F }}$ 
confirm that the prescription does not meet the required data, have difficulty understanding the medical prescription, and indicate that medical prescriptions do not have legible letters and clear. $36.36 \%$ of Q. F. do not update the pharmacotherapeutic profile and $54.55 \%$ do not perform verification and monitoring of the patient's location. $45.55 \%$ of the Q. F., indicate that the single dose boxes are not correctly identified and $18.18 \%$ do not review and corroborate the medication deposited in each box. $27.27 \%$ of Q. F., indicate that the prepared dose of a patient has been insufficient in 24 hours. And 54.55\% have noticed insufficient pharmaceutical products that in an emergency and / or emergency, and only $36.36 \%$ of Q. F. have a medicine box or other reserve products for each patient. $27.27 \%$ of Q.F. confirm that there is no strict control at the time of delivery of medications and other products; and 36.36\%, for narcotics. $36.36 \%$ of Q.F. confirm that the administration of the medication is not given at the exact time. Confirming by $54.55 \%$, who have observed difficulties in meeting the time of administration of the unit dose, ratifying with $63.64 \%$ that does not observe strict compliance, in terms of the frequency of administration of the drug to each patient. Conclusions: The existence of difficulties in the dispensing process per unit dose was verified according to compliance with the Technical Standard of the Ministry of Health. This limits the good dispensing practices per unit dose at the Zacarías Correa Valdivia Regional Hospital in Huancavelica.

KEYWORDS: Pharmaceutical chemist, good practices, dispensation and unit dose.

\section{INTRODUCCIÓN}

El trabajo presentado aborda las buenas prácticas de dispensación en dosis unitaria del Hospital Regional Zacarías Correa Valdivia de Huancavelica. Se destaca la importancia de una adecuada y correcta práctica, en cuanto al proceso que se requiere para lograr exitosamente la terapia medicamentosa en los pacientes internados en el hospital.

El interés de este trabajo viene dado, ya que se trata de un área clínica de suma importancia, donde el trabajo central del profesional químico farmacéutico, conjuntamente a otros profesionales sanitarios, influye en gran medida en la rehabilitación del paciente.

Existen procedimientos que demandan mayor cuidado, como es el sistema de distribución de medicamentos por dosis unitaria, ya que la población principal son los pacientes hospitalizados (1), este método consiste en la dispensación de las dosis unitarias para 24 horas, en forma individualizada por paciente y en donde además el químico farmacéutico realiza el seguimiento.

Cabe resaltar, que la dispensación de medicamentos por dosis unitaria es una forma terapéutica, con gran eficacia, esto debido, a que se le ofrece la administración exacta, control y seguimiento individualizado en un tiempo organizado, a cada paciente hospitalizado. Garantizando de esta manera, la recuperación del paciente por medio del tratamiento farmacoterapeutico.
Por lo expuesto, la estrategia terapéutica por dosis unitaria, alcanza un valor muy alto dentro del rubro de salud, en tanto, hacer de su funcionalidad adecuada y correcta, seguirá sumando a garantizar la atención integral de salud de todos los habitantes del país (2). La Organización Mundial de la Salud (OMS) hace referencia la importancia de la Atención Farmacéutica para mejorar la calidad de vida del paciente y mejorar los resultados de salud (3).

Las buenas prácticas de dispensación son el acto farmacéutico asociado a una adecuada y correcta, entrega y distribución de medicamentos, con las consecuentes prestaciones específicas, como el análisis de la orden médica, la información clara sobre el medicamento en cuanto a su uso y conservación, la preparación de las dosis a administrar y, además, la aplicación del medicamento cuando lo amerite (4); con la finalidad de contribuir al seguimiento farmacoterapéutico de los pacientes.

Es importante resaltar que son varios estudios los que manifiestan, que el uso y empleo inapropiado de los medicamentos, actualmente son un problema de salud pública, y que genera consecuencias a nivel social y económico.

Sin duda alguna, la praxis del profesional químico farmacéutico, y su desarrollo adecuado, influye directamente la rehabilitación del paciente, y errores en este proceso, podrían ser contraproducentes y tener consecuencias negativas. Por ello es necesario mejoras las debilidades de las competencias del profesional químico farmacéutico y su equipo de trabajo, en 
cuanto a la dispensación de medicamentos por dosis unitaria.

La dispensación de medicamentos por dosis unitaria, es un método de dispensación y control de la medicación en servicios de salud organizados y coordinados por la farmacial, es decir, nos referimos a un nosocomio, específicamente el servicio de hospitalización, donde se involucra la cantidad física de un medicamento indicado por el profesional prescriptor como una dosis de tratamiento para un paciente, cuyo envase permite la administración segura y directa al paciente a una determinada hora, ofreciendo la oportunidad para efectuar un adecuado seguimiento a la terapia medicamentosa del paciente (5).

Es importante reconocer que el medicamento, como combinación de uno o más fármacos con otras sustancias, es capaz de producir efectos o cambios fisiológicos en el paciente y es preciso resaltar que sus efectos pueden ser tanto benéficos en la recuperación del estado de salud, como también perjudiciales, al utilizarse inapropiadamente; misma confirmada por la Organización Mundial de la Salud, donde manifiesta que los efectos adversos de los medicamentos están entre las 10 causas princípiales de muerte en el mundo. Motivo por el cual, el uso y empleo inapropiado de estos medicamentos en la actualidad, se han convertido un problema de salud pública (6).

El presente estudio es uno de los primeros realizados en la región de Huancavelica que persiguen las buenas prácticas de dispensación por dosis unitaria, asimismo, busca captar e identificar las debilidades que el Hospital Regional Zacarias Correa Valdivia presenta, en este proceso; para buscar medidas de solución que genere la eficiencia y eficacia en este rubro.

El objetivo del presente trabajo fue determinar las buenas prácticas de dispensación por dosis unitaria Hospital Regional Zacarías Correa Valdivia de Huancavelica, basadas en la norma técnica del Ministerio de Salud del año 2009, sobre el sistema de dispensación de medicamentos en dosis unitaria para los establecimientos del Sector Salud.

\section{MATERIAL Y METODOS}

Investigación de tipo descriptivo, teniendo como finalidad observar, conocer y describir las prácticas de dispensación por dosis unitaria del Hospital Regional Zacarías Correa Valdivia de Huancavelica.

El diseño es no experimental, transversal y descriptivo, debido a que no se van a manipular variables, y va ser evaluado en un solo momento.

La población está integrada por los profesionales que laboran en el Hospital Zacarías Correa Valdivia, específicamente por 11 profesionales químicos farmacéuticos.

Todos los datos obtenidos fueron bajo la técnica de encuesta, se diseñó y valido por juicio de expertos el instrumento BPDDUMP, para la la presente investigación, Buenas Prácticas De Dispensación En Dosis Unitaria Del Hospital Regional Zacarias Correa Valdivia De Huancavelica 2019. Los profesionales que colaboraron en la misma, son la Dra. Q.F. Diana Esemeralda Andamayo Flores, la Mg. Q.F. Lizzy Jeanette Mendoza Gutierrez y Mg. Q.F. Martha Raquel Valderrama Sueldo.

\section{RESULTADOS}

De los 11 (100.00 \%) Químicos Farmacéuticos, 11 $(100.00 \%)$ conocen las partes de una receta que exige la norma técnica de salud; $9(81.82 \%)$ confirman que en el hospital los medicamentos de control estricto se prescriben en un receta separada, mientras que $2(18.18 \%)$ no lo confirman; 9 (81.82\%) refieren que la receta mal elaborada, lo devuelve al médico, mientras que $2(18.18 \%)$ no lo refiere; asimismo $5(45.56 \%)$ confirman que la prescripción médica en el hospital, cumple con todos los datos requeridos, mientras que $6(54.55 \%)$ no lo confirman (Grafico 1).

De los 11 (100.00 \%) Químicos Farmacéuticos, $7(63.64 \%)$ confirman que el contenido de la prescripción médica es claro, mientras que $4(36.36 \%)$ no lo confirman; $6(54.55 \%)$ refiere que la mayoría de veces, tiene dificultades para comprender el contenido receta médica, mientras que $5(45.45 \%)$ no lo refiere; $5(45.45 \%)$ refieren que las recetas médicas tienen letra legible y clara, mientras que $6(54.55 \%)$ no lo refiere; asimismo $3(27.27 \%)$ refieren que si no logra comprender alguna parte de la receta médica, utiliza la deducción para lograr interpretarla, mientras que 8 $(72.73 \%)$ no lo refieren (gráfico 2$)$. 
La receta mal elaborada, lo devuelves al médico

Confirma que, en este hospital, los medicamentos de control estricto se.

Confirma que la prescripción médica en el hospital, cumple con todos los datos..

Conoce las partes de una receta que exige la norma técnica de salud

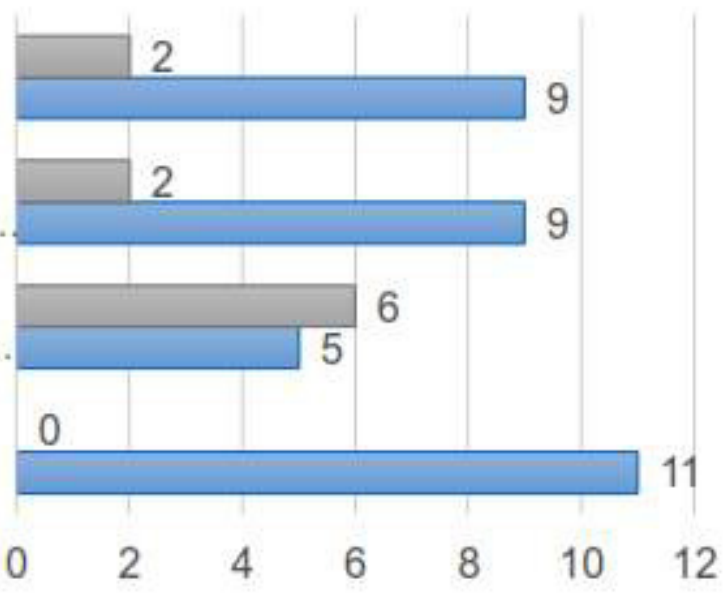

$\square$ No $\square$ SI

Gráfico 1. Validación de la prescripción médica.

Si no logra comprender alguna parte de la receta médica, usted utiliza la...

Las recetas médicas que usted recepciona, tienen letra legible y clara

La mayoría de veces, tiene dificultades para comprender el contenido receta..

Confirma que el contenido de la prescripción médica es claro

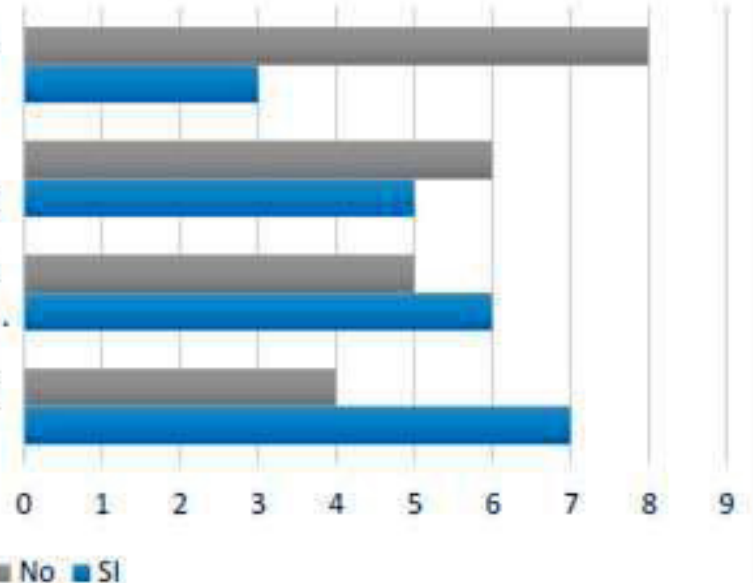

Gráfico 2. Interpretación de la información contenida en la orden médica.

Actualiza a diario el perfil farmacoterapeutico del paciente tratante en hospitalización

Desarrolla el perfil farmacoterapeutico del paciente hospitalizado

Conoce el procedimiento para realizar un perfil farmacoterapeutico

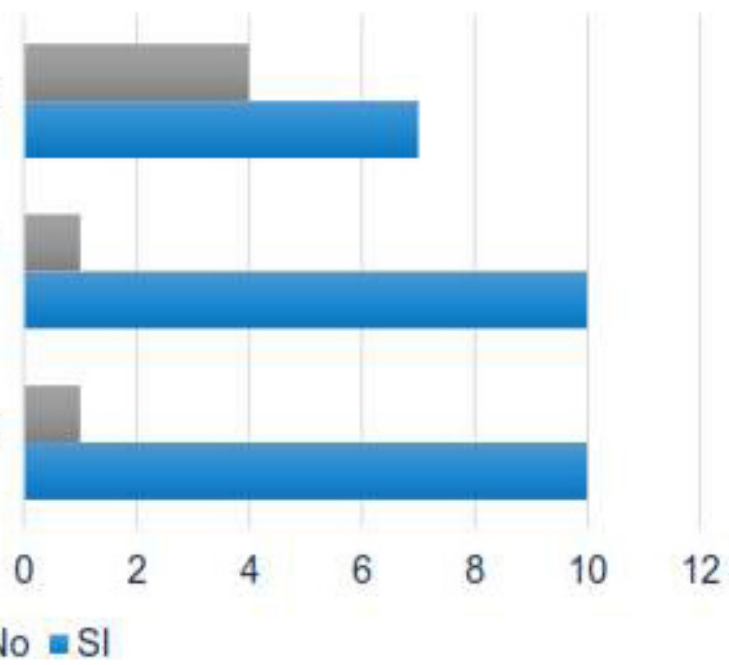

Gráfico 3. Elaboración del perfil farmacoterpeútico 
De los 11 (100.00 \%) Químicos Farmacéuticos, 10 $(90.91 \%)$ refiere que conoce el procedimiento para realizar un perfil farmacoterapeutico, mientras que $1(9.09 \%)$ no lo conoce; $10(90.91 \%)$ refieren que desarrolla el perfil farmacoterapeutico del paciente hospitalizado, mientras que 1 (9.09\%) no lo desarrolla; asimismo 7 (63.64\%) refieren que actualizan a diario el perfil farmacoterapeutico del paciente tratante en hospitalización, mientras que $4(36.36 \%)$ no lo actualizan (grafico 3).

De los 11 (100.00 \%) Químicos Farmacéuticos, $11(100.00 \%)$ refiere que registra el número de dosis entregadas para las 24 horas de cada paciente; asimismo $5(45.45 \%)$ refieren que verifica y hace seguimiento de la ubicación del paciente, como el traslado de servicio, alta o muerte, mientras que 6 $(54.55 \%)$ no lo realizan (gráfico 4$)$.
De los 11 (100.00 \%) Químicos Farmacéuticos, 10 $(90.91 \%)$ confirma que los cajetines de medicamentos, están claramente identificados con el nombre del paciente, número de historia clínica, numero de cama y el servicio al que debe llevarse, mientras que 1 (9.09 \%) no lo confirma; asimismo 6 (54.55\%) refieren que los cajetines de la unidosis, están correctamente identificados con los datos del paciente, mientras que $5(45.45 \%)$ no lo refieren (gráfico 5).

De los 11 (100.00 \%) Químicos Farmacéuticos, $9(81.82 \%)$ refieren que revisan y corroboran la medicación depositada en cada cajetín, conforme al perfil farmacoterapeutico del paciente, mientras que 2 (18.18\%) no lo realiza (gráfico 6).

De los 11 (100.00 \%) Químicos Farmacéuticos, $8(72.73 \%)$ confirman que la cantidad de dosis
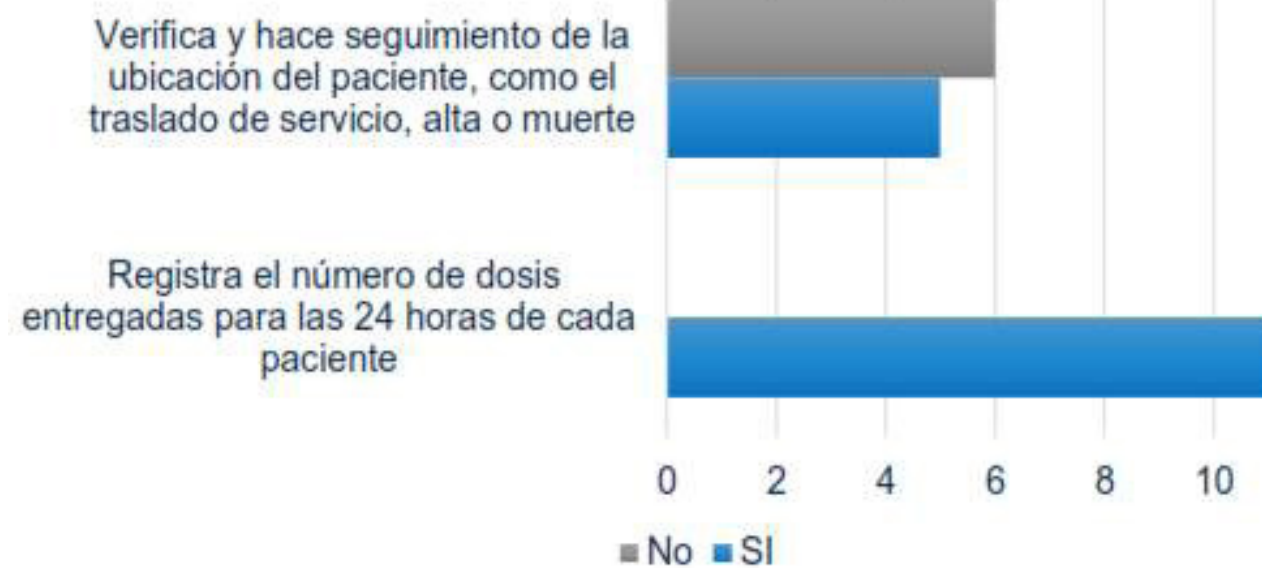

Gráfico 4. Seguimiento del perfil farmacoterapeutico.
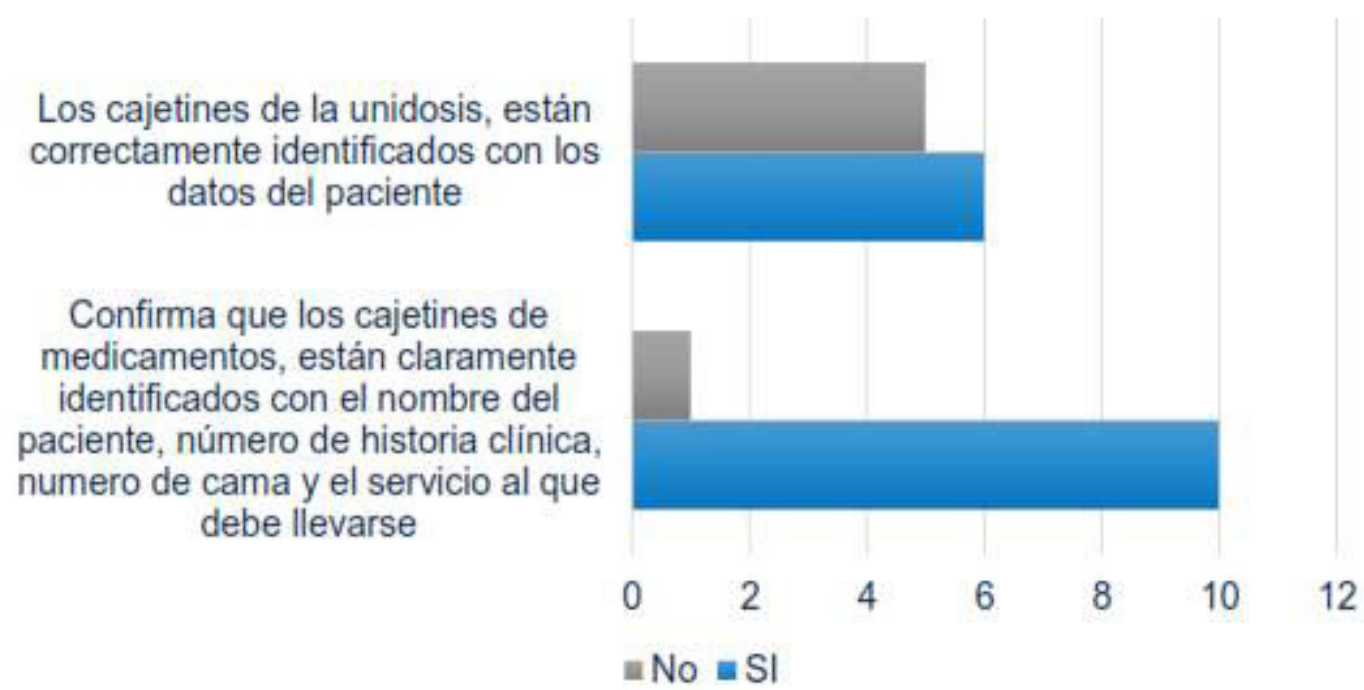

Gráfico 5. Los cajetines deben estar identificados con los datos del paciente. 


\section{Revisa y corrobora la medicación depositada en cada cajetín, conforme al perfil farmacoterapeutico del paciente}

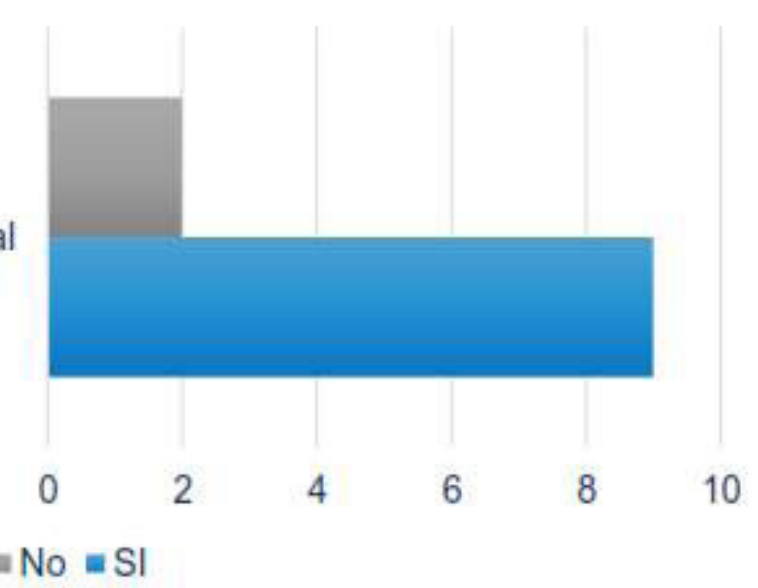

Gráfico 6. Ratificación de la medicación depositada en cada cajetín.
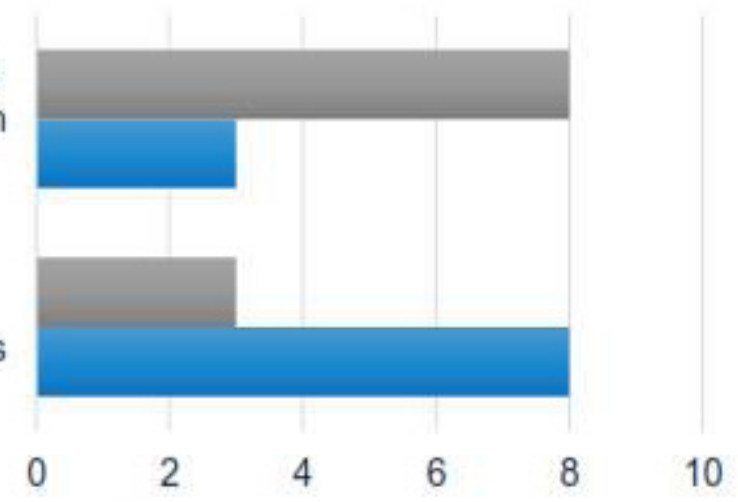

\section{Ha observado que la dosis preparada de un paciente, ha sido insuficiente en 24 horas \\ Confirma que la cantidad de dosis preparada en este hospital, es suficiente para un periodo de 24 horas de tratamiento de cada paciente}

\section{$=\mathrm{No} \equiv \mathrm{SI}$}

Gráfico 7. Cantidad suficiente para un periodo de 24 horas de tratamiento.

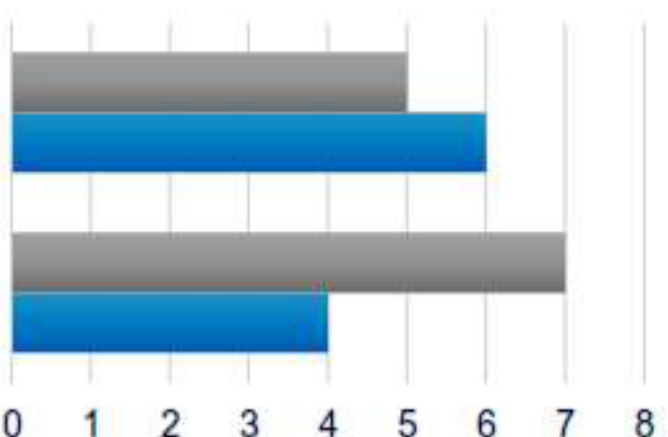

$\approx \mathrm{No}=\mathrm{SI}$

Gráfico 8. Cajetines de medicamentos por paciente.

preparada en este hospital, es suficiente para un periodo de 24 horas de tratamiento de cada paciente, mientras que 3 (27.27\%) no lo confirma; 3 (27.27\%) han observado que la dosis preparada de un paciente, ha sido insuficiente en 24 horas; mientras que 8 (72.73 \%) no lo han observado (gráfico 7).
De los 11 (100.00\%) Químicos Farmacéuticos, $6 \quad(54.55 \%)$ consideran que en una urgencia y/o emergencia, se ha notado insuficiencia de los productos farmacéuticos, mientras que $5(45.45 \%)$ no lo consideran; 4 (36.36\%) consideran que cuenta con un cajetín de medicamentos u otros productos ya 


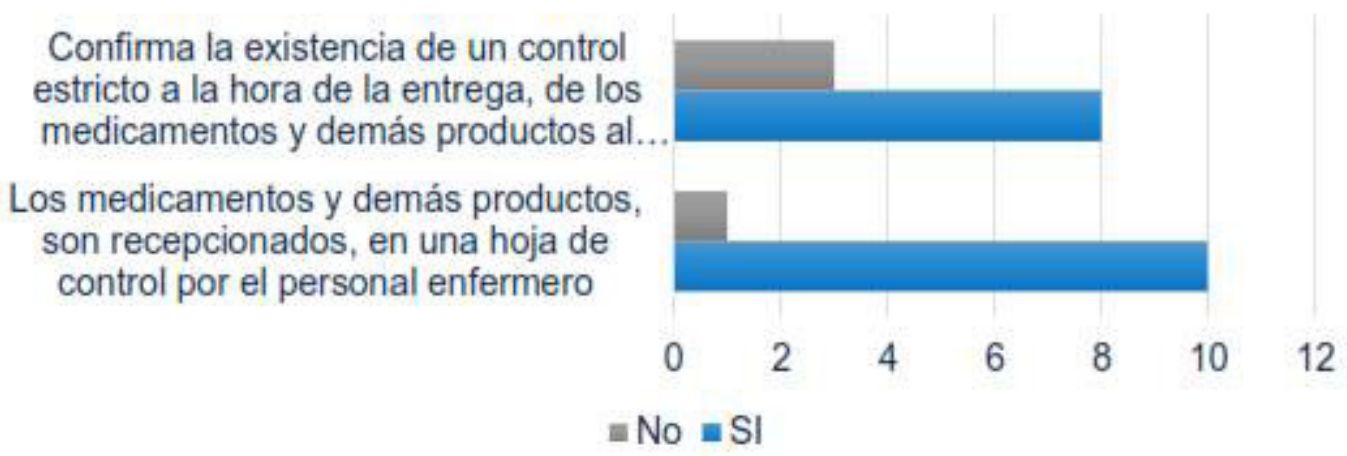

Gráfico 9. El personal enfermero recepciona con total control los medicamentos.

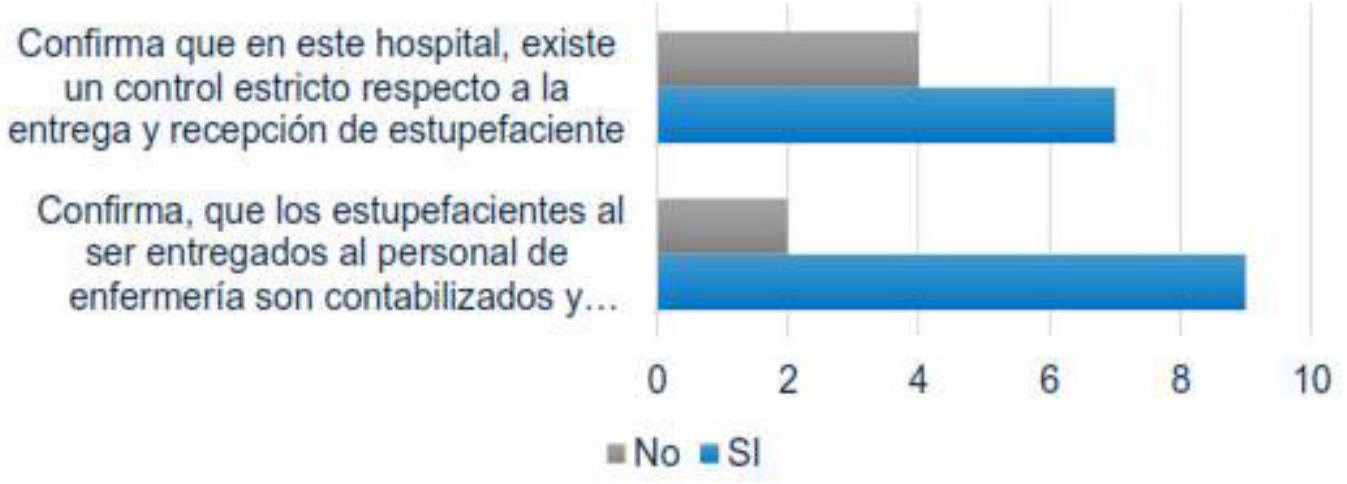

Gráfico 10. Los estupefacientes tienen mayor control.

preparados, de reserva por cada paciente; mientras que $7(63.64 \%)$ no lo consideran (gráfico 8).

De los 11 (100.00\%) Químicos Farmacéuticos, $10(90.91 \%)$ refieren que los medicamentos y demás productos, son recepcionados, en una hoja de control por el personal enfermero, mientras que $1(9.09 \%)$ no lo refieren; $8(72.73 \%)$ confirman la existencia de un control estricto a la hora de la entrega, de los medicamentos y demás productos al personal enfermero; mientras que $3(27.27 \%)$ no lo confirman (gráfico 9).

De los $11(100.00 \%)$ Químicos Farmacéuticos, $9 \quad(81.82 \%)$ confirman que los estupefacientes al ser entregados al personal de enfermería son contabilizados y guardados en un botiquín, donde pueda ser supervisado, mientras que $2(18.18 \%)$ no lo confirman; 7 (63.64\%) confirman que en este hospital, existe un control estricto respecto a la entrega y recepción de estupefaciente; mientras que 4 (36.36 $\%$ ) no lo confirman (gráfico 10).

De los 11 (100.00 \%) Químicos Farmacéuticos, $10(90.91 \%)$ valida los documentos necesarios, antes de administrar los medicamentos a cada paciente, mientras que $1(9.09 \%)$ no lo validan; 8 (72.73\%) verifican que el personal enfermero revisa previamente la tarjeta de control u hoja de administración, antes de administrar el medicamento a cada paciente; mientras que $3(27.27 \%)$ no lo verifican (grafico 11$)$.

De los 11 (100.00\%) Químicos Farmacéuticos, $7(63.64 \%)$ confirman que en este hospital la administración del medicamento se da en el horario exacto, ya establecido por el médico tratante, mientras que $4(36.36 \%)$ no lo confirman; $6(54.55 \%)$ han observado dificultades en el cumplimiento de la hora de administración de las unidosis; mientras que 5 (45.45 $\%)$ no lo han observado; 5 (45.45\%) verifican que el enfermero administre los medicamentos a los pacientes, mientras que $6(54.55 \%)$ no lo verifican; 4 (36.36 \%) han observado el cumplimiento estricto, en cuanto la frecuencia de administración del medicamento a cada paciente; mientras que 7 (63.64 $\%$ ) no lo han observado (gráfico 12).

\section{DISCUSIÓN}

El sistema de distribución de medicamentos en dosis unitarias, representa una estrategia terapéutica importante, consiste en un sistema científico diseñado, 


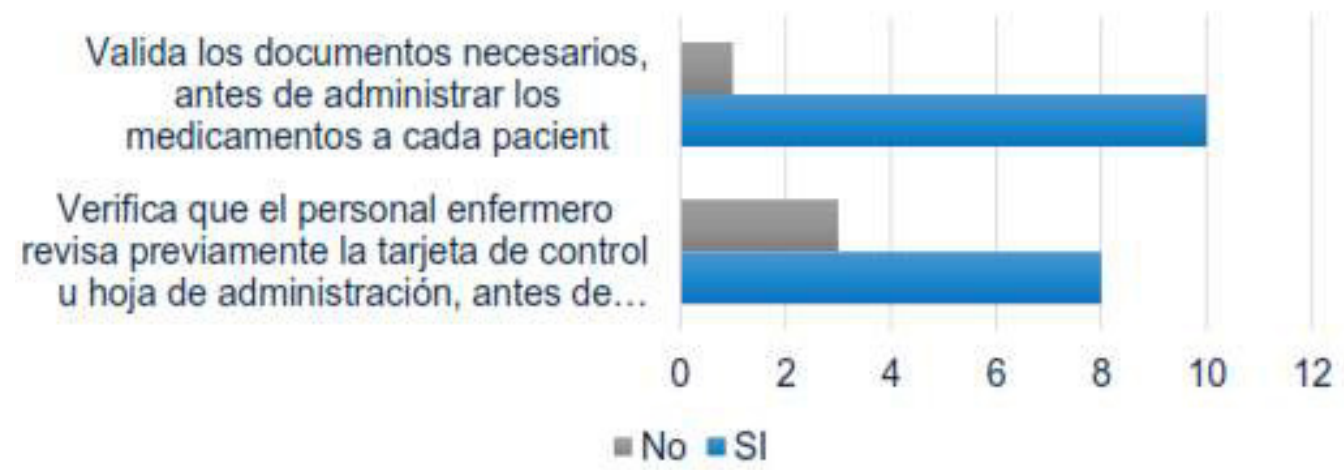

Gráfico 11. Revisar previamente la tarjeta de control de inventario u hoja de administración.

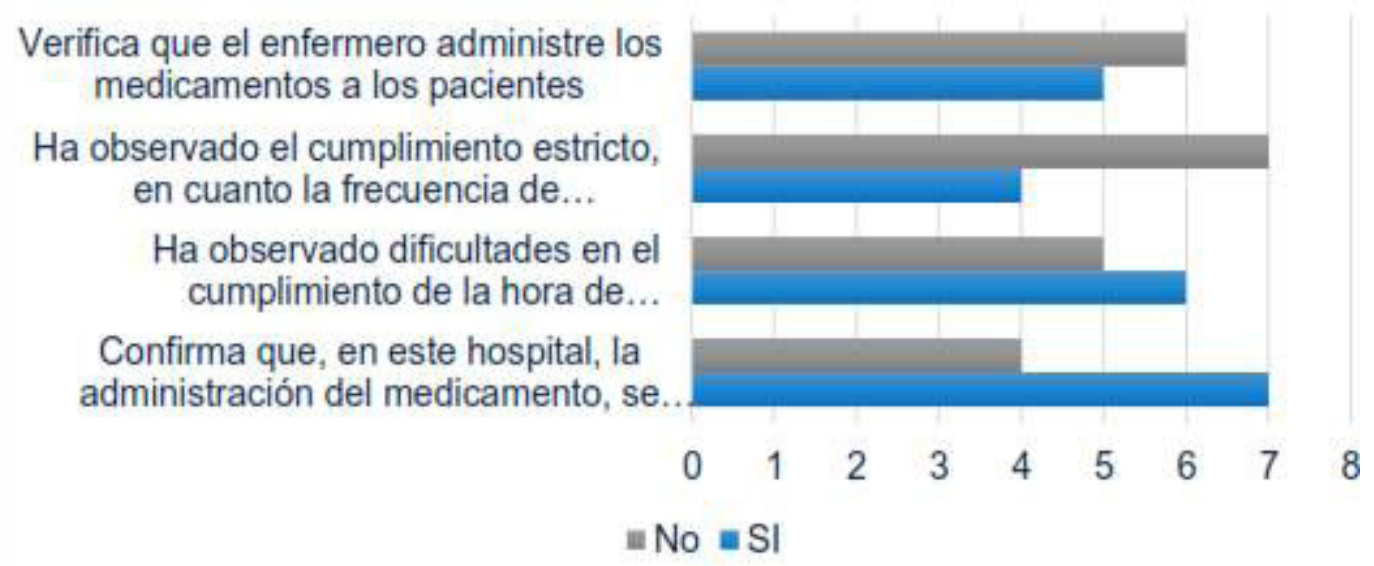

Gráfico 12. Administración del medicamento en el horario y frecuencia establecidos

que mejora la seguridad de los pacientes y la calidad de la terapéutica en los hospitales (5). Reconociendo esta acepción, recalcamos la importancia y el valor de este sistema; y nos es, mucho más sustancial, ya que, el Perú atiende una gran taza de pacientes, 872.283 pacientes con morbilidad de hospitalización durante el periodo del 2018 según el Repositorio Único Nacional de Información en Salud (REUNIS) (7).

Asimismo, se ha notado que la farmacoterapia no siempre tiene el éxito esperado en tiempo y en efectividad (5), esta situación puede ser resultados de muchos factores. Un sujeto determinante en la práctica de este proceso, es el químico farmacéutico, de quien depende junto con otros profesionales de la salud, que este proceso se lleve a cabo adecuada y eficazmente. También un factor que suma a esta problemática es la falta de recursos humanos, perjudicando a cabalidad el cumplimiento de las buenas prácticas de dispensación por dosis unitaria (8).

El 100\% de los Químicos Farmacéuticos del Hospital Regional Docente Materno Infantil "El
Carmen" y Hospital Nacional "Ramiro Prialé Prialé" de Huancayo - ESSALUD, y el 78\% de Químicos Farmacéuticos del Hospital Regional Docente Clínico Quirúrgico "Daniel Alcides Carrión", respondieron que si creen que las habilidades y competencias como Químico Farmacéutico, inciden en las buenas prácticas de dispensación en dosis unitaria (9).

Validando el planteamiento de este estudio, en cuanto a la importancia del Q.F. en el proceso de las buenas prácticas de dispensación por dosis unitaria.

Conforme a la praxis, en cuanto a la dispensación por dosis unitaria en el Hospital Regional Zacarías Correa Valdivia, se demostró que en el camino entre el cumplimiento de la prescripción médica hasta la administración del medicamento, hay dificultades, que limitan la correcta utilización del fármaco. Una problemática que merece ser atendida, ya que los errores de medicación y sus consecuencias negativas constituyen en la actualidad un grave problema de salud pública, con importantes repercusiones, desde el punto de vista humano, asistencial y económico 
(10), por lo que, es importante poner énfasis en los resultados siguientes, del Hospital Regional Zacarías Correa Valdivia de Huancavelica (2019).

En cuanto al cumplimiento de la prescripción médica, fue alarmante que el $54.55 \%$ de profesionales químicos farmacéuticos del Hospital Regional Zacarías Correa Valdivia de Huancavelica, confirme que la prescripción no cumpla con los datos requeridos. Al igual que se observó en el estudio de Carriel donde, los errores detectados en la prescripción de los servicios del el Hospital Sagrado Corazón de Jesús fluctúan entre 84 \% y 89,43 \% (11), validando los resultados de este estudio.

Asimismo, los químicos farmacéuticos de Hospital Regional Zacarías Correa Valdivia en un $54.55 \%$ indican que tienen dificultades para comprender la receta médica; el mismo porcentaje refieren que las recetas médicas no tienen letra legible y clara. El $84 \%$ de recetas médicas tienen datos inconformes del paciente y médico, también se reconoció que el $99 \%$ de medicamentos prescriptos están con datos inconformes (12). Asimismo, en los estudios de Salva R. y Torres A. (2019) se encontraron resultados en cuanto a los errores de prescripción la más frecuente, la omisión de vía de administración en un $32.58 \%$ (8), corroborando los resultados de la presente investigación.

Cabe resaltar que los profesionales del Hospital Regional Zacarías Correa Valdivia de Huancavelica, en un $100 \%$ conocen las partes de una receta que exige la norma técnica de salud; lo que sugiere que el personal está actualizado, la Organización Mundial de la Salud destaca el importante papel que desempeña este profesional sanitario por sus conocimientos y por situarse como el más accesible a la población3; además el $81.82 \%$ confirman que en el hospital los medicamentos de control estricto se prescriben en un receta separada; asimismo el $81.82 \%$ refiere que la receta mal elaborada, lo devuelve al médico.

La prescripción médica, es un documento de suma importancia, y el primer paso para desarrollar el proceso de dispensación, que finalmente tendrá lugar durante el proceso de preparación, dispensación y administración del medicamento, como lo indica Rita Moreira Silva en su artículo, Errores de Prescripción (13). La prescripción médica debe sujetarse a lo establecido en la legislación vigente Decreto Supremo No021-2001 -SA.18, ya que de esta dependerá, todo el proceso de la farmacoterapia y por tanto está en juego la recuperación del paciente; que habiendo errores en la misma, conllevara a consecuencias potencialmente lesivos para los pacientes (13).

En cuanto al seguimiento farmacoterapeutico, los profesionales químicos farmacéuticos de este hospital, manifiestan que en un $36.36 \%$, que no actualizan el perfil farmacoterapeutico del paciente tratante en hospitalización. Asimismo que el $54.55 \%$, no realiza la verificación y seguimiento de la ubicación del paciente, como el traslado de servicio, alta o muerte. Son muchas los estudios que reafirman la deficiente práctica de este indicador como la investigación de Carriel donde en un $100 \%$ no existe un llenado del perfil farmacoterapéutico para los pacientes (11), por tanto, no hay seguimiento farmacoterapéutico. También en el estudio de Patín se encontró que en un $11 \%$ de perfiles farmacoterapéuticos están transcritos con inconformidades (12).

Paucarhuanca en su estudio, mostró que el seguimiento farmacoterapéutico se dio en menos del $1,5 \%$ de los pacientes (14), igualmente, Córdova y Jiménez. indicaron que lleva a cabo el seguimiento farmacoterapéutico en $2,6 \%$ en la Clínica Internacional (15). Por ultimo Blanco, indica que uno de los errores encontrados es la falta de seguimiento farmacoterapéutico, en Hospital de Niños Dr. José Manuel De Los Ríos (16), aceptando y reafirmando los resultados obtenidos en la presente investigación a.

De acuerdo a la experiencia profesional, y por estos datos observados, es una actividad regularmente omitida en este establecimiento; sin embargo, es un procedimiento sumamente importante, ya que permite reconocer posibles errores en la dosis, duplicidad de prescripción, posibles interacciones, devolución de medicamentos, de manera que hay mayor control de la medicación; que finalmente contribuirá al bienestar de los pacientes. Girón y D’Alessio recomiendan su implementación, en su Guía Sistema de distribución de medicamentos por dosis unitarias (1)

Otro indicador importante en este proceso, es el llenado en envases seguros e identificados para la dispensación, los profesionales químicos farmacéuticos de este hospital en un $45.55 \%$ refieren que los cajetines de la unidosis, no están correctamente identificados con los datos del paciente. Corroborado por las investigaciones de Córdova y Jiménez, quienes encontraron errores de dispensación en un $2,4 \%$ en 
la Clínica Internacional (15), asimismo, Blanco en el Hospital de Niños Dr. José Manuel De Los Ríos, concluye la existencia de errores de dispensación (16).

De la misma forma, en un $18.18 \%$ de profesionales Q.M. del Hospital Zacarías Correa Valdivia no revisan y corroboran la medicación depositada en cada cajetín, conforme al perfil farmacoterapeutico del paciente.

En lo que refiere a la dosis de cada paciente, los resultados del Hospital Regional Zacarías Correa Valdivia, indica que más de la cuarta parte (27.27 $\%)$ de profesionales químicos farmacéuticos de este nosocomio, han observado que la dosis preparada de un paciente, ha sido insuficiente en 24 horas. Según la Sociedad Española de Farmacia Hospitalaria - SELT (2003), el sistema de dispensación por dosis unitaria, debe ofrecer la oportunidad para efectuar un adecuado seguimiento a la terapia medicamentosa del paciente durante 24 horas de tratamiento (4), al mismo tiempo, que más de la mitad (54.55\%) confirman que en una urgencia y/o emergencia, se ha notado insuficiencia de los productos farmacéuticos, reafirmando el mismo problema; y solo el $36.36 \%$ confirman que cuentan con un cajetín de medicamentos u otros productos ya preparados, de reserva por cada paciente.

El desabastecimiento de medicamentos en un hospital del estado, es realmente una barrera al acceso a la salud (17), y magnamente perjudicial en la intervención adecuada de los pacientes hospitalizados; situación que de alguna manera obliga a la población acudir y las empresas privadas, provocando mayor costes, siendo el rubro de salud el mayor coste en la familia peruana como lo indica Ministerio de Salud (18). Además cabe señalar, que la región de Huancavelica se encuentra en el segundo grupo de pobreza más alta en Perú al 2018 (19), teniendo mayores limitaciones al acceso de servicios de salud a nivel particular.

Con relación a la recepción y control, el $27.27 \%$ de profesionales químicos farmacéuticos no confirman la existencia de un control estricto a la hora de la entrega, de los medicamentos y demás productos al personal enfermero. Además el $36.36 \%$, de los mismos indican que no existe un control estricto respecto a la entrega y recepción de estupefacientes. Este rubro está regido por el Reglamento de estupefacientes, psicotrópicos y otras sustancias sujetas a fiscalización sanitaria (20), la importancia de esta se encuentra en que un estupefaciente como sustancia psicotrópica, tiene alto potencial de producir conducta abusiva y/o dependencia (psíquica/física), manifestado por la Administración Nacional, Alimentos y Tecnología Médica (21), indicador que puede ser influenciado por el deficiente traslado de los medicamentos por parte del químico farmacéutico (22).

En cuanto a la administración del medicamento, los profesionales químicos farmacéuticos de este nosocomio en un $27.27 \%$ no verifican que el personal enfermero revise previamente la tarjeta de control u hoja de administración, antes de administrar el medicamento a cada paciente.

El incumplimiento de esta actividad, hace más proclive a errores en cuanto a la terapia medicamentosa, que ya expuestos, no solo provoca un déficit en cuanto a la recuperación, sino generar reacciones adversa, que van contra la salud y vida del paciente.

Molina et a., reportan tasas de incidencia de acontecimientos adversos de admisiones hospitalarias que fluctúan entre un $2,9 \%$ a un $16,6 \%$; y de cuyos acontecimientos adversos, entre el $25 \%$ y $50 \%$ se consideraron prevenibles, y finalmente entre un 5\% y un $13 \%$ de acontecimientos adversos resultaron en la muerte de los pacientes (5). Córdova y Jiménez muestran indicadores de reacciones adversas a medicamentos de seguridad notificadas en 10 casos en la Clínica Internacional (15).

Asimismo, el $36.36 \%$ de Q.F. del Hospital Zacarias Correa Valdivia de Huancavelica indica que la administración del medicamento no se da en el horario exacto, ya establecido por el médico tratante. Confirmando estos datos, se sabe que el $54.55 \%$ de químicos farmaceuticos han observado dificultades en el cumplimiento de la hora de administración de las unidosis, así como el $63.64 \%$ no observan el cumplimiento estricto, en cuanto la frecuencia de administración del medicamento a cada paciente. Igualmente el estudio de Blanco en el Hospital de Niños Dr. José Manuel De Los Ríos, donde verifico deficiencias en el suministro de medicamentos (12).

La principal característica del sistema de dosis unitaria, según American Society of Hospital Pharmacists, está en que, el tratamiento de cierta dosis de medicación, permita la administración segura y directa al paciente a una determinada hora (4), cuyo fin es contribuir a la recuperación eficaz y el menor tiempo posible el estado de salud del paciente. El 
incumplimiento de esta regla, va contra la misma estrategia terapéutica, provocando deficiencia para la recuperación del paciente.

En último lugar, Moreira manifiesta que conocer los tipos de errores en el proceso de dispensación, resulta indispensable para maximizar la prevención (13). En tanto se ha podido observar las principales dificultades y errores en cuanto a la práctica de dispensación en dosis unitaria reconocida en el Hospital Regional Zacarías Correa Valdivia de Huancavelica.

\section{CONCLUSIONES}

Se verifico la existencia de dificultades en el proceso de dispensación por dosis unitaria según al cumplimiento de la Norma técnica del Ministerio de Salud. Lo que limita las buenas prácticas de dispensación por dosis unitaria en el Hospital Regional Zacarías Correa Valdivia de Huancavelica. En consideración al cumplimiento de la prescripción médica, en un porcentaje mayor a la mitad (54.55\%) de Q.M., confirmaron que la prescripción no cumple con los datos requeridos. El mismo porcentaje indica que tienen dificultades para comprender la receta médica; y que las recetas médicas no tienen letra legible y clara.

En consideración a contribuir al seguimiento farmacoterapéutico de pacientes del servicio de hospitalización según criterios específicos; se resalta que mayor a la cuarta parte $(36.36 \%)$ de Q. F. no actualizan el perfil farmacoterapeutico. Además un porcentaje mayor a la mitad $(54.55 \%)$, no realiza la verificación y seguimiento de la ubicación del paciente, como el traslado de servicio, alta o muerte.

En consideración a los envases debidamente identificados para la dispensación, se revelo que casi la mitad $(45.55 \%)$ de los Q. F., manifestaron que los cajetines de la unidosis, no están correctamente identificados con los datos del paciente. $Y$ en cuanto a la ratificación de la medicación depositada en cada cajetín, se observa que el en un mínimo porcentaje $(18.18 \%)$ de profesionales no revisan y corroboran la medicación depositada en cada cajetín, conforme al perfil farmacoterapeutico del paciente.

Acerca de las cantidades exactas para un periodo de 24 horas. El 27.27 \% de Q. F., han observado que la dosis preparada de un paciente, ha sido insuficiente en 24 horas. Y un porcentaje mayor a la mitad (54.55 \%) confirmaron que en una urgencia y/o emergencia, se ha notado insuficiencia de los productos farmacéuticos, y solo el $36.36 \%$ de Q. F. confirman que cuentan con un cajetín de medicamentos u otros productos ya preparados, de reserva por cada paciente.

En cuanto a la recepción y control de la dosis indicada, aproximadamente la cuarta parte $(27.27 \%)$ de Q.F. confirman que no existe un control estricto a la hora de la entrega, de los medicamentos y demás productos al personal enfermero. También, el $36.36 \%$, indica que no existe un control estricto respecto a la entrega y recepción de estupefacientes.

En cuanto al cumplimiento con el rol para la administración de la dosis, aproximadamente la cuarta parte $(27.27 \%)$ no verifica que el personal enfermero revise previamente la tarjeta de control u hoja de administración, antes de administrar el medicamento a cada paciente, el $36.36 \%$ de Q.F. indican que la administración del medicamento no se da en el horario exacto. Confirmando por el $54.55 \%$, quienes han observado dificultades en el cumplimiento de la hora de administración de las unidosis, ratificando con el $63.64 \%$ que no observa el cumplimiento estricto, en cuanto la frecuencia de administración del medicamento a cada paciente.

\section{Correspondencia}

Lizzy Jeanette Mendoza Gutierrez

Av. Giráldez 542, Huancayo 12001. Junin, Perú

\section{REFERENCIAS BIBLIOGRÁFICAS}

1. Giron N; D’Alessio R. Guía para el desarrollo de servicios farmacéuticos hospitalarios: logística del suministro de medicamentos. Washington: Organización Panamericana de la Salud;1997.

2. Ministerio de Salud. Misión y visión. Perú : DIGEMID; 2019. (Citado el 15 de noviembre del 2019) Disponible en: http://www.digemid.minsa.gob. pe/Main.asp?Seccion=40

3. Portalfarma.com. Cumplimiento terapeutico. Madrid: Consejo General de Colegios Oficiales de Farmaceuticos; 2017. (Citado el 15 de noviembre del 2019) Disponible en: https://www.portalfarma.com/ Ciudadanos/saludpublica/consejosdesalud/Paginas/ cumplimientoterapeutico.aspx

4. American Society of Hospital Pharmacists. ASHP statement on unit dose drug distribution. Am J Hosp Pharm. 1989; 46:176.

5. Molina E, Ramos Y, Guerrero B. Sistema de distribución de medicamentos en dosis unitarias 
y errores de medicación. Educación y salud boletín científico de la salud del ICSa. 2018; 7:131-5.

6. Organización Mundial de la Salud. Perspectivas políticas de la OMS sobre medicamentos La farmacovigilancia: garantía de seguridad en el uso de los medicamentos. Ginebra: Organización Mundial de la Salud; 2004. (Citado el 15 de noviembre del 2019) Disponible en: https://apps.who.int/ medicinedocs/pdf/s6166s/s6166s.pdf

7. Ministerio de Salud. Perú: REUNIS-Repositorio Único Nacional de Información en Salud. Lima: Ministerio de Salud; 2017 (Citado el 15 de noviembre del 2019) Disponible en: https://www.minsa.gob. pe/reunis/recursos_salud/MORBE002017.asp

8. Salva K, Torres Y. Evaluación de las buenas prácticas de dispensación del sistema de distribución de medicamentos en dosis unitaria en el Hospital De Apoyo "Manuel Ángel Higa Arakaki" - Satipo. Tesis. Huancayo: Universidad Privada De Huancayo "Franklin Roosevelt"; 2019.

9. Cerron G, Romero M. Rol del farmacéutico en la dispensación de dosis unitaria en hospitales de la provincia Huancayo. Tesis. Huancayo: Universidad Privada De Huancayo "Franklin Roosevelt"; 2017.

10. Hurtado J. Factores asociados a la implementación del sistema de dispensación/distribución de medicamentos por dosis unitaria, en el Hospital del Instituto Ecuatoriano de Seguridad Social Babahoyo 2013-2016: Tesis. Lima:Universidad Nacional Mayor De San Marcos; 2017.

11. Carriel K. Evaluación del Sistema de Distribución de Medicamentos por Dosis Unitaria (SDMDU) en el Hospital Sagrado Corazón de Jesús de la ciudad de Quevedo y propuesta de plan de mejoras. Tesis. Ecuador: Universidad Central del Ecuador; 2019.

12. Patín R. Evaluación del sistema de distribución de medicamentos por dosis unitaria implementado en el Servicio de Medicina Interna del Hospital General Alfredo Noboa Montenegro, Guaranda provincia de Bolívar. Tesis. Riobamba: Escuela Superior Politécnica de Chimborazo;2018.

13. Moreira R. Errores de Prescripción. Barcelona: CEDIMCAT;2018. (Citado el 15 de noviembre del 2019) Disponible en: https://www.cedimcat.info/ index.php?option $=$ com_content $\&$ view $=$ article \&id $=192$ :errores-de-prescripcion-ejemplos-de-erroresde-prescripcion-frecuentes-y-su-posible-prevencion \&catid $=47$ : seguridad $\&$ lang $=$ es

14. Paucarhuanca K. Evaluación del Sistema de Dispensación de Medicamentos mediante Dosis Unitaria en el Hospital nivel II Carlos Tuppia García Godos - EsSalud de Ayacucho 2016. Tesis. Ayacucho: Universidad Nacional San Cristóbal De Huamanga; 2017.
15. Córdova L, Jiménez A. Sistema de Distribución de Medicamentos en Dosis Unitaria (SDMDU) en la Clínica Internacional, sede Lima, julio- diciembre 2012. Tesis. Lima: Universidad Norbert Wiener; 2013

16. Blanco O. Implementación de un Sistema de Distribución de Medicamentos por Dosis Unitaria (SDMDU) para el Servicio Médico de Nefrologia del Hospital de Niños Dr. José Manuel de los Ríos. Tesis. Venezuela: Universidad Central de Venezuela; 2015.

17. Alarcón L. Desabastecimiento de medicamentos: una barrera al acceso de la salud. Lima: La República; 2019.

18. Ministerio de Salud. Manual De Buenas Prácticas De Dispensación. Norma técnica de salud: sistema de dispensación de medicamentos en dosis unitaria para los establecimientos del Sector Salud. Lima: Ministerio de Salud; 2009 (Citado el 15 de noviembre del 2019) Disponible en: http://gestionensalud. medicina.unmsm.edu.pe/wp-content/ uploads/2015/08/MS RB 061022 DIGEMID58. pdf

19. Instituto Nacional de Estadística e Informática. InformeTécnico Evolución de la pobreza monetaria 2007 - 2018. Lima: Instituto Nacional de Estadística e Informática; 2018 (Citado el 15 de noviembre del 2019) Disponible en: https://www.inei.gob.pe/media/ MenuRecursivo/publicaciones_digitales/Est/Lib146/ libro.pdf

20. Ministerio de Salud. Decreto Supremo No 023-2001SA Reglamento de Estupefacientes Psicotrópicos Sujetas a Fiscalización Sanitaria.Lima: DIGEMID: 2001. (Citado el 15 de noviembre del 2019) Disponible en: http://www.digemid.minsa.gob.pe/UpLoad/Up Loaded/PDF/DecretoSupremoN023-2001-SA.pdf

21. Administración Nacional, Alimentos y Tecnología Médica. ¿Qué Son Los Psicotrópicos Y Estupefacientes? Buenos Aires: Administración Nacional, Alimentos y Tecnología Médica; 2011 (Citado el 15 de noviembre del 2019) Disponible en:http://www.anmat.gov.ar/Medicamentos/ psicotropicos_y_estupefacientes.pdf

22. Chupayo N,Bastidas S. Evaluación del funcionamiento del sistema de distribución de medicamentos en dosis unitaria en el Hospital Regional Docente Materno Infantil El Carmen. Tesis. Huancayo: Universidad Privada De Huancayo "Franklin Roosevelt"; 2017.

\begin{tabular}{|c|}
\hline Recibido: $14 / 02 / 2020$ \\
Aceptado: 13/06/2020 \\
\hline
\end{tabular}

